\title{
The Signless Laplacian Spectral Radius of Unicyclic and Bicyclic Graphs with a Given Girth *
}

\author{
$\mathrm{Ke} \mathrm{Li}^{1} \quad$ Ligong $\mathrm{Wang}^{2 \dagger} \quad$ Guopeng $\mathrm{Zhao}^{3}$ \\ Department of Applied Mathematics \\ Northwestern Polytechnical University \\ Xi'an, Shaanxi 710072, P. R. China. \\ ${ }^{1}$ like2007happy@163.com \\ ${ }^{2}$ ligongwangnpu@yahoo.com.cn \\ ${ }^{3}$ zgp081632@sina.com
}

Submitted: Dec 30, 2010; Accepted: Sep 6, 2011; Published: Sep 20, 2011

Mathematics Subject Classifications: 05C50, 15A18.

\begin{abstract}
Let $\mathcal{U}(n, g)$ and $\mathcal{B}(n, g)$ be the set of unicyclic graphs and bicyclic graphs on $n$ vertices with girth $g$, respectively. Let $\mathcal{B}_{1}(n, g)$ be the subclass of $\mathcal{B}(n, g)$ consisting of all bicyclic graphs with two edge-disjoint cycles and $\mathcal{B}_{2}(n, g)=\mathcal{B}(n, g) \backslash \mathcal{B}_{1}(n, g)$. This paper determines the unique graph with the maximal signless Laplacian spectral radius among all graphs in $\mathcal{U}(n, g)$ and $\mathcal{B}(n, g)$, respectively. Furthermore, an upper bound of the signless Laplacian spectral radius and the extremal graph for $\mathcal{B}(n, g)$ are also given.
\end{abstract}

Keywords: Unicyclic graphs; Bicyclic graphs; Signless Laplacian spectral radius; Girth

\section{Introduction}

Throughout the paper, let $G=(V, E)$ be a connected undirected simple graph with $V=V(G)=\left\{v_{1}, v_{2}, \cdots, v_{n}\right\}$ and $E=E(G)=\left\{e_{1}, e_{2}, \cdots, e_{m}\right\}$. The order of a graph is the cardinality of its vertex set. Especially, if $m=n$ or $m=n+1$, then $G$ is called a unicyclic or bicyclic graph, respectively. The girth $g=g(G)$ of $G$ is the length of the shortest cycle in $G$. Denote by $d(u, v)$ the distance between the vertices $u$ and $v$ of $G$, which is the length of the shortest path joining the vertex $u$ with $v$. Suppose that

\footnotetext{
*Supported by the National Natural Science Foundation of China (No.11171273, No.10871158), the Natural science Foundation of Shaanxi Province (No.SJ08A01), and SRF for ROCS, SEM.

${ }^{\dagger}$ Corresponding author
} 
$U \subseteq V(G), u \in V(G)$, and $u \notin U$, the distance between $u$ and $U$, denoted by $d(u, U)$, is the minimum distance between $u$ and a vertex in $U$. Let $\Delta=\Delta(G)$ be the maximum degree of $G$. Let $A(G)$ and $D(G)$ be the adjacency matrix and diagonal matrix of vertex degree of $G$, respectively. The Laplacian matrix of $G$ is $L(G)=D(G)-A(G)$ and the signless Laplacian matrix of $G$ is $Q(G)=D(G)+A(G)$. The Laplacian spectral radius $\lambda_{1}(G)$ is the largest eigenvalue of $L(G)$ and the signless Laplacian spectral radius or $Q$ spectral radius $q_{1}(G)$ is the largest eigenvalue of $Q(G)$. Moreover, if $G$ is connected, by the Perron-Frobenius Theorem, we have that $Q$-spectral radius is simple and has a unique unit positive eigenvector. We refer to such an eigenvector as Perron vector of $G$.

The adjacency matrix $A(G)$ and Laplacian matrix $L(G)$ are studied extensively and the main results are referred to [1] and [14], respectively. Recently, the problem about determining the extremal graphs with the maximal signless Laplacian spectral radius for a class of graphs attracts people's attention. Some properties of signless Laplacian spectra of graphs and some possibilities for developing the spectral theory of graphs based on $Q(G)$ are discussed in $[3,4,5]$. Fan and Yang studied the signless Laplacian spectral radius of graphs with a given number of pendent vertices in [9]. Feng and Yu studied the signless Laplacian spectral radius of unicyclic graphs with a given number of pendent vertices or independence number in [7]. Liu, Tan and Liu studied the (signless) Laplacian spectral radius of unicyclic and bicyclic graphs with $n$ vertices and $k$ pendent vertices in [12]. Zhai, Yu and Shu determined the extremal graph with the maximal Laplacian spectral radius among all bicyclic graphs with a given girth in [15]. In this paper, we determine the unique graph with maximal signless Laplacian spectral radius among all unicyclic and bicyclic graphs with a given girth $g$, respectively. Furthermore, the upper bound of the signless Laplacian spectral radius and the the extremal graph for all bicyclic graphs with a given girth $g$ are also obtained.

Let $\mathcal{U}(n, g)$ and $\mathcal{B}(n, g)$ be the set of unicyclic and bicyclic graphs on $n$ vertices with a fixed girth $g$, respectively. Denote by $\mathcal{B}_{1}(n, g)$ the subclass of $\mathcal{B}(n, g)$ consisting of all bicyclic graphs with two edge-disjoint cycles and by $\mathcal{B}_{2}(n, g)=\mathcal{B}(n, g) \backslash \mathcal{B}(n, g)$ consisting of bicyclic graphs with three pairwise internal disjoint paths $P_{p+1}, P_{q+1}, P_{r+1}$ with common endpoints. Let $P_{n}$ (resp. $C_{n}$ ) be the path (cycle) on $n$ vertices. Denote by $B_{p, q}^{k}$ the graph obtained from two disjoint cycles $C_{p}$ and $C_{q}$ by identifying a vertex $u$ of $C_{p}$ with a vertex $v$ of $C_{q}$ and attaching $k$ pendent edges to $u(v)$. Denote $P_{p, q, r}^{k}$ the graphs consisting of three pairwise internal disjoint paths $P_{p+1}, P_{q+1}, P_{r+1}$ with common endpoints and $k$ pendent edges at one of the common endpoints, which is shown in Figure 1.

\section{Preliminaries}

Let $G$ be a bicyclic graph. The the base of $G$, denoted by $B(G)$, is the minimal bicyclic subgraph of $G$. Clearly, $B(G)$ is the unique bicyclic subgraph of $G$ containing no pendent vertices, and $G$ can be obtained from $B(G)$ by planting trees to some vertices of $B(G)$. A hanging tree of $v$ in $G$, denoted by $T(v)$, is a rooted tree with $v$ as its root vertex. For a better classification, in the following discussion, a vertex set of a hanging tree does not include its root vertex. 

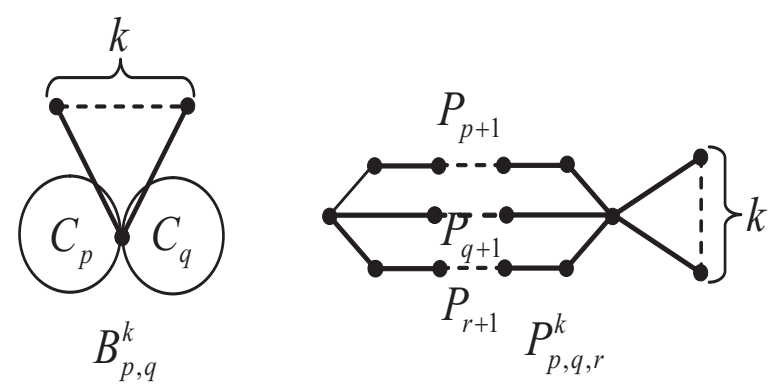

Figure 1: The bicyclic graphs $B_{p, q}^{k}$ and $P_{p, q, r}^{k}$.

Bicyclic graphs have two types of bases which are shown in Figure 2. Denote by $B(p, l, q)$ the graph obtained by joining a new path $u_{1} u_{2} \cdots u_{l}$ between two vertex-disjoint cycles $C_{p}$ and $C_{q}$, where $u_{1} \in V\left(C_{p}\right)$ and $u_{l} \in\left(C_{q}\right)$. In particular, $B(p, 1, q) \cong C_{p} u v C_{q}$ for $u \in V\left(C_{p}\right)$ and $u \in V\left(C_{q}\right)$, where $C_{p} u v C_{q}$ denotes the graph obtained from $C_{p}$ and $C_{q}$ by identifying a vertex $u$ of $C_{p}$ with a vertex $v$ of $C_{q}$. Denote by $P(p, q, r)$ the graph consisting of three pairwise internal disjoint paths $P_{p+1}, P_{q+1}, P_{r+1}$ with common endpoints, that is, $P(p, q, r) \cong P_{p, q, r}^{0}$.

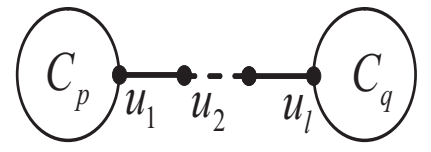

$B(p, l, q)$

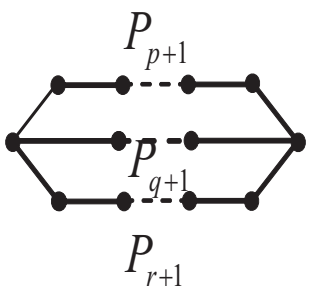

$P(p, q, r)$

Figure 2: The bases of bicyclic graphs.

Clearly, $\mathcal{B}_{1}(n, g)$ and $\mathcal{B}_{2}(n, g)$ can also be defined as follows:

$\mathcal{B}_{1}(n, g)=\{G \in \mathcal{B} \mid B(G)=B(p, l, q)$ for some $l \geq 1$ and $p, q \geq 3\}$.

$\mathcal{B}_{2}(n, g)=\{G \in \mathcal{B} \mid B(G)=P(p, q, r)$ for some $p, q, r \geq 1\}$.

Lemma 2.1. [13] Let $G$ be a graph on $n$ vertices. Then $q_{1}(G) \leq \max \left\{d_{u}+m_{u}: u \in\right.$ $V(G)\}$, where $m_{u}=\left(\sum_{u v \in E(G)} d_{v}\right) / d_{u}$ is the average degree of neighbors of $u$, the equality holds if and only if $G$ is regular or semi-regular bipartite.

Lemma 2.2. [11] Let $G$ be a connected graph and $u, v$ be the two vertices of $G$. Suppose $v_{1}, v_{2}, \cdots, v_{s} \in N(v) \backslash\{N(u) \cup u\}\left(1 \leq s \leq d_{v}\right)$ and $G^{*}$ is the graph obtained from $G$ by deleting the edges $v v_{i}$ and adding $u v_{i}(1 \leq i \leq s)$. Let $X=\left(x_{1}, x_{2}, \cdots, x_{n}\right)^{t}$ be the 
principal eigenvector of $Q(G)$, where $x_{i}$ corresponds to $v_{i}(1 \leq i \leq n)$. If $x_{u} \geq x_{v}$, then $q_{1}(G)<q_{1}\left(G^{*}\right)$.

Now we consider the graph $G_{u v}$ obtained from a connected graph $G$ by subdividing the edge $u v$, that is, by replacing edge $u v$ with edges $u w, w v$, where $w$ is an additional vertex. We call the following two types of paths internal paths: (a) A sequence of vertices $v_{0}, v_{1}, \cdots, v_{k}(k \geq 2)$, where $v_{0}, v_{1}, \cdots, v_{k}$ are distinct and $v_{0}=v_{k+1}$ of degree at least 3 , $d_{v_{i}}=2$, for $i=1,2, \cdots, k$ and $v_{i-1}, v_{i}(i=1,2, \cdots, k+1)$ are adjacent; (b) A sequence of vertices $v_{0,1}, \cdots, v_{k}(k \geq 0)$ such that $v_{i-1}, v_{i}$ are adjacent, $d_{v_{0}} \geq 3, d_{v_{k+1}} \geq 3$ and $d_{v_{i}}=2$ where $1 \leq i \leq k$.

Lemma 2.3. [3, 8] Let $G$ be a connected graph and $u v$ be some edge on the internal path of $G$ as we defined above. If we subdivide $u v$, that is, substitute it by $u w, w v$ with a new vertex $w$, and denote the new graph by $G_{u v}$, then $q_{1}\left(G_{u v}\right)<q_{1}(G)$.

Lemma 2.4. [3] Suppose $G$ is a nontrivial simple and connected graph. Let $v$ be some vertex of $G$. For nonnegative integers $k, l$, let $G(k, l)$ denote the graph obtained from $G$ by adding pendant paths of length $k, l$ at $v$. If $k \geq l \geq 1$, then $q_{1}(G(k, l))>q_{1}(G(k+1, l-1))$.

Lemma 2.5. [6] Let $G$ be a connected graph. Suppose $v_{1}, v_{2}$ are vertices each of degree at least 3 and $v_{1} v_{2}$ is an edge of $G$. Let $G^{*}$ be the connected graph obtained from $G$ by contracting $v_{1} v_{2}$, that is, deleting the edge and identifying vertices $v_{1}, v_{2}$. Then $q_{1}(G)<q_{1}\left(G^{*}\right)$.

Lemma 2.6. [2] Let $G$ be a graph on $n$ vertices with at least an edge and the maximum degree of $G$ be $\Delta$. Then we have $q_{1}(G) \geq \Delta+1$. The equality holds if only if $G$ is a star.

Lemma 2.7. [13] Let $G$ be a simple and connected graph on $n$ vertices, its degree sequence is $d_{v_{1}}, d_{v_{2}}, \cdots, d_{v_{n}}$. Then we have

(1) $q_{1}(G) \leq \max \left\{\frac{d_{u}\left(d_{u}+m_{u}\right)+d_{v}\left(d_{v}+m_{v}\right)}{d_{u}+d_{v}}: u v \in E\right\}$.

(2) $q_{1}(G) \leq \max \left\{d_{u}+d_{v}: u v \in E\right\}$.

Lemma 2.8. [10] Let $G$ be a connected graph. Suppose $v_{1}, v_{k}$ are vertices each of degree at least 3 and $N\left(v_{1}\right) \cap N\left(v_{k}\right)=\emptyset$. Suppose further that the unique path $P=v_{1} v_{2} \cdots v_{k}$ from $v_{1}$ to $v_{k}$ is an internal path. Let $G^{\prime}$ be a connected graph obtained from $G$ by collapsing the entire internal path (i.e., delete all edges $v_{1} v_{2}, v_{2} v_{3}, \cdots, v_{k-1} v_{k}$ and identify the vertices $\left.v_{1}, v_{2}, \cdots, v_{k}\right)$. Then $q_{1}(G)<q_{1}\left(G^{\prime}\right)$.

Lemma 2.9. [6] Let $G$ be a connected graph and $P$ be a pendant path in $G$. Suppose $e$ is an edge in $P$ and $G^{*}$ is the graph obtained from $G$ by subdividing $e$, then we have $q_{1}(G)<q_{1}\left(G^{*}\right)$.

\section{Main Results}

In this section, the extremal graphs with the maximal signless Laplacian spectral radius among all graphs in $\mathcal{U}(n, g)$ and $\mathcal{B}(n, g)$ and the upper bound of the signless Laplacian spectral radius for $\mathcal{B}(n, g)$ will be presented. 
Theorem 3.1. For every pair of positive integers $n, g$ with $3 \leq g \leq n-1$, the graph $U_{g, n-g}$ is the extremal graph with the maximal signless Laplacian spectral radius among all unicyclic graphs with a given girth $g$, where the graph $U_{g, n-g}$ is obtained from the cycle $C_{g}$ by attaching $n-g$ pendent edges to some vertex of the cycle.

Proof. For any unicyclic graph $G \in \mathcal{U}(n, g)$, we only need to consider the trees attached to some vertices on the cycle $C_{g}$ of $G$. Firstly, if there exists at least one vertex on the hanging trees having degree at least 3 , we choose the vertex $w$ which has the minimal value $d\left(w, C_{g}\right)$ among all such vertices described above, then by Lemma 2.8 collapse the entire internal path joining the vertex $w$ with the the vertex on the cycle $C_{g}$, then by Lemma 2.9 we get a new graph $G^{\prime}$ by subdividing some pendent edges several times to keep the order of $G^{\prime}$ the same as the order of $G$. Through these edges operations above, $G^{\prime}$ has a larger signless Laplacian spectral radius and smaller number of the vertices with degree at least 3 on the trees. Continuing the operations as above, we can get all trees attached consist of pendent paths. Secondly, by Lemma 2.2 comparing the eigencomponents corresponding to the root vertices attached by pendent paths and reattaching all the pendent paths to the root vertex corresponding to the largest eigencomponent. Finally, by Lemma 2.4, all pendent paths with the length equal to or greater than 2 are transformed into pendent edges with the same number of vertices as the order of all the original pendent paths, which results in a graph with a larger signless Laplacian spectral radius. Then the proof of the Theorem 3.1 is completed.

Theorem 3.2. For every pair of positive integers $n, g$ with $3 \leq g \leq \frac{n+1}{2}, B_{g, g}^{n-2 g+1}$ is the extremal graph with the maximal signless Lapalcian spectral radius among all graphs in $\mathcal{B}_{1}(n, g)$.

Proof. As the discussion of the proof of Theorem 3.1, we know that the graph $G^{*}$ with the maximal signless Laplacian spectral radius among all graphs in $\mathcal{B}_{1}(n, g)$ is obtained from $B\left(G^{*}\right)$ by attaching some pendent edges to some vertex $v$ of $B\left(G^{*}\right) . B\left(G^{*}\right)$, as we define above, has the same form as $B(p, l, q)$, where a path $P=u_{1} u_{2} \cdots u_{l}$ joining the vertex $u_{1}$ on the cycle $C_{g}$ with the vertex $u_{l}$ on the cycle $C_{q}$ for $l \geq 1$. Firstly, it suffices to show $u_{1}=u_{l}=u$, namely $l=1$. Assume to the contrary that $l \geq 2$. By Lemmas 2.4 and 2.6, when we delete the whole path $P$ and identify all the vertices into a vertex $u$, then by Lemmas 2.9 and 2.4 , we will get a graph $G^{\prime}$ with $q_{1}\left(G^{\prime}\right) \geq$ $q_{1}\left(G^{*}\right)$, where $G^{\prime}$ is obtained by attaching $n-(g+q)+1$ pendent edges to some vertex $v$ of $B(g, 1, q)$. This is a contradiction. Secondly, we can versify that $q=g$ in the graph $G^{*}$. If $q \geq g+1$, then by Lemma 2.3 we can transform the cycle $C_{q}$ into a cycle having the same length $g$, then by Lemmas 2.9 and 2.4 we can get a graph $G^{\prime}$ with $q_{1}\left(G^{\prime}\right) \geq q_{1}\left(G^{*}\right)$. This is a contradiction. Finally, by Lemma 2.2 , we show $u=v$. Assume that $u \neq v$. Let $N(u)=\left\{w_{1}, w_{2}, \cdots, w_{t}, \cdots, w_{d_{u}}\right\}$, let $t$ be the number of pendant vertices of the neighbors of $u, N(v)=\left\{s_{1}, s_{2}, \cdots, s_{d_{v}}\right\}, s_{1}, s_{2} \in V\left(C_{p}\right)$ and $d_{v}=4$. If $x_{u} \geq x_{v}$, then we can get a new graph $G^{\prime}=G^{*}-\left\{v s_{1}, v s_{2}\right\}+\left\{u s_{1}, u s_{2}\right\}$ with $q_{1}\left(G^{*}\right)<q_{1}\left(G^{\prime}\right)$. This is a contradiction. If $x_{v}>x_{u}$, then we also can get a new graph $G^{\prime \prime}=G^{*}-\left\{u w_{1}, u w_{2}, \cdots, u w_{t}\right\}+\left\{v w_{1}, v w_{2}, \cdots, v w_{t}\right\}$ with $q_{1}\left(G^{*}\right)<q_{1}\left(G^{\prime \prime}\right)$. This is also a contradiction. Hence the proof of Theorem 3.2 is completed. 
Theorem 3.3. For every pair of positive integers $n, g$ with $3 \leq g \leq \frac{2(n+1)}{3}, P_{\lfloor g / 2\rfloor,\lceil g / 2\rceil,\lceil g / 2\rceil}^{n-\lceil 3 g / 2\rceil}$ is the extremal graph with the maximal signless Laplacian spectral radius among all graphs in $\mathcal{B}_{2}(n, g)$.

Proof. We discuss the two facts as follows:

Fact 1. Let $G^{*}$ have the maximal signless Laplacian spectral radius among all graphs in $\mathcal{B}_{2}(n, g)$. When $n \geq\lceil 3 g / 2\rceil-1$, then $G^{*}$ is the graph obtained from $P(\lfloor g / 2\rfloor,\lceil g / 2\rceil,\lceil g / 2\rceil)$ by attaching $n-\lceil 3 g / 2\rceil+1$ pendent edges to a unique vertex.

Proof of Fact 1. The proof need distinguish two case that $g$ is even or odd.

Case 1. We firstly consider the case that $g$ is even. Set $a=g / 2$ and suppose that $B\left(G^{*}\right)=P(p, q, r)$, where $p \leq q \leq r$ and $p+q=2 a$. It suffices to show that $p=q=r=a$. Note $p+q+r \geq 3 a$ and $n \geq(p+q+r)-1$.

If $n=3 a-1$, then $G^{*}$ can not contain any pendent vertices and $p+q+r=3 a$. Since $p+q=2 a, r=a$ and hence $p \leq q \leq a$. If $p \leq a-1$, then $q \geq a+1$, which contradicts to $q \leq a$. Thus $p=q=r=a$.

If $n=3 a$, then $p+q+r \leq 3 a+1$. Since $p+q=2 a, r \leq a+1$. This implies that $q \leq a+1$. So $(p, q, r) \in\{(a, a, a),(a, a, a+1),(a-1, a+1, a+1)\}$. Assume, for a contradiction, that $(p, q, r) \in\{(a, a, a+1),(a-1, a+1, a+1)\}$, then $G^{*}$ can not contain any pendent edges, in other words, $G^{*}$ is isomorphic to one of $P(a, a, a+1)$ and $P(a-1, a+1, a+1)$. When $a=2$, straightforward calculations show that $q_{1}(P(2,2,3))=4.932, q_{1}(P(1,3,3))=5$ and $q_{1}\left(P_{2,2,2}^{1}\right)=5.5141$. So we have $\max \left\{q_{1}(P(2,2,3)), q_{1}(P(1,3,3))\right\}<q_{1}\left(P_{2,2,2}^{1}\right)$, a contradiction. When $a \geq 3, G^{*}$ can not contain a pair of adjacent 3 -vertices. So if by Lemma 2.7,

$$
q_{1} \leq \max \left\{d_{u}+d_{v}: u v \in E(G)\right\}=5 .
$$

However, $q_{1}\left(P_{a, a, a}^{1}\right)>\Delta+1=5$ since in this case $G^{*}$ is not a star. Thus $q_{1}\left(G^{*}\right)<q_{1}\left(P_{a, a, a}^{1}\right)$, a contradiction. Therefore, $p=q=r=a$.

Now it remains the case $n \geq 3 a+1$. If $r=a$, clearly $p=q=a$ since $p+q=2 a$ and $p \leq q \leq a$. Next we suppose that $r \geq a+1$, and set $k=n-(p+q+r)+1$, namely the number of pendent vertices in $G^{*}$. Then $k \leq n-3 a$. If $k$ is fixed, then we can find that $\max \left\{d_{u}+m_{u} \mid u \in V(G)\right\}$ attains the maximal value just when $p=1$ and $k$ pendent edges are incident to a 3 -vertex of $P(p, q, r)$. In this case,

$$
\max \left\{d_{u}+m_{u} \mid u \in V(G)\right\}=k+3+\frac{k+7}{k+3}=k+4+\frac{4}{k+3} .
$$

By Lemma 2.7, we have

$$
q_{1}\left(G^{*}\right)<\max \left\{d_{u}+m_{u} \mid u \in V(G)\right\}=k+4+\frac{4}{k+3}
$$

since in this case $G^{*}$ can not neither be regular nor semi-regular. Note that $k+4+\frac{4}{k+3}$ is increasing with nonnegative integer $k$. Thus

$$
q_{1}\left(G^{*}\right)<n-3 a+4+\frac{4}{n-3 a+3} \leq n-3 a+5
$$


since $n \geq 3 a+1$. However, by Lemma $2.1, q_{1}\left(P_{a, a, a}^{n-3 a+1}\right)>\Delta+1=n-3 a+5$, a contradiction.

Case 2. Now we consider the case that $g$ is odd. The proof is similar to Case 1 . Suppose that $B\left(G^{*}\right)=P(p, q, r)$, where $p \leq q \leq r$ and $p+q=g$. It suffices to show that $p=\lfloor g / 2\rfloor=\frac{g-1}{2}, q=r=\lceil g / 2\rceil=\frac{g+1}{2}$. Note that $p+q+r \geq g+\frac{g+1}{2}=\frac{3 g+1}{2}$ and $n \geq \frac{3 g+1}{2}-1$.

If $n=\frac{3 g+1}{2}-1$, then $G$ can not contain any pendent edges and $p+q+r=\frac{3 g+1}{2}$. Since $p+q=g, r=\frac{g+1}{2}$ and $p \leq q \leq \frac{g+1}{2}$. If $p \leq \frac{g-1}{2}$, then $q \geq \frac{g+1}{2}+1$, which contradicts to $q \leq r$. So the only case is that $p=\lfloor g / 2\rfloor=\frac{g-1}{2}, q=\lceil g / 2\rceil=\frac{g+1}{2}$ and $r=\lceil g / 2\rceil=\frac{g+1}{2}$.

If $n=\frac{3 g+1}{2}$, then $p+q+r \leq \frac{3 g+1}{2}+1$. Since $p+q=g, r \leq \frac{g+1}{2}+1$. This implies that $q \leq$ $\frac{g+3}{2}$ and $p \geq \frac{g-3}{2}$ for $g \geq 5$. So $(p, q, r) \in\left\{\left(\frac{g-1}{2}, \frac{g+1}{2}, \frac{g+1}{2}\right),\left(\frac{g-1}{2}, \frac{g+1}{2}, \frac{g+3}{2}\right),\left(\frac{g-3}{2}, \frac{g+3}{2}, \frac{g+3}{2}\right)\right\}$. Moreover, if $(p, q, r) \in\left\{\frac{g-1}{2}, \frac{g+1}{2}, \frac{g+3}{2}\right),\left(\frac{g-3}{2}, \frac{g+3}{2}, \frac{g+3}{2}\right\}$, then $G^{*}$ can not contain any pendent edges, in other words, $G^{*}$ is isomorphic to one of $P\left(\frac{g-1}{2}, \frac{g+1}{2}, \frac{g+3}{2}\right)$ and $P\left(\frac{g-3}{2}, \frac{g+3}{2}, \frac{g+3}{2}\right)$. When $g=3$, the conclusion holds clearly. When $g=5$, straightforward calculations show that $q_{1}\left(P\left(\frac{g-1}{2}, \frac{g+1}{2}, \frac{g+3}{2}\right)\right)=q_{1}(2,3,4)=4.7728, q_{1}\left(P\left(\frac{g-3}{2}, \frac{g+3}{2}, \frac{g+3}{2}\right)\right)=$ $q_{1}(1,4,4)=4.9032, \quad q_{1}\left(P_{\lfloor g / 2\rfloor,\lceil g / 2\rceil,\lceil g / 2\rceil}^{1}\right)=q_{1}\left(P_{2,2,2}^{1}\right)=5.3552$. So we have that $\max \left\{q_{1}(2,3,4), q_{1}(1,4,4)\right\}<q_{1}\left(P_{2,2,2}^{1}\right)$, a contradiction. When $g \geq 7, G^{*}$ can not contain a pair of adjacent 3-vertices. So by Lemma 2.7,

$$
q_{1}\left(G^{*}\right) \leq \max \left\{d_{u}+d_{v} \mid u v \in E(G)\right\}=5 .
$$

However, $q_{1}\left(P_{\frac{g-1}{2}, \frac{g+1}{2}, \frac{g+1}{2}}^{1}\right)>\Delta+1=5$ since $P_{\frac{g-1}{2}, \frac{g+1}{2}, \frac{g+1}{2}}^{1}$ is not a star, a contradiction. Therefore $p=\lfloor g / 2\rfloor=\frac{g-1}{2}, q=r=\lceil g / 2\rceil=\frac{g+1}{2}$.

Now it remains the case that $n \geq \frac{3 g+1}{2}+1$. If $r=\frac{g+1}{2}$, clearly, $p=\frac{g-1}{2}, q=\frac{g+1}{2}$ since $p+q=g$ and $p \leq q \leq r$. Next we suppose $r \geq \frac{g+1}{2}+1$ and set $k=n-(p+q+r)+1$, namely the number of pendent vertices in $G^{*}$. Then $k \leq n-\frac{3 g+1}{2}$. We can find $\max \left\{d_{u}+m_{u} \mid u \in\right.$ $V(G)\}$ attains the maximal value just when $g=3$, namely $p=1$, and $k$ pendent edges are incident to a 3 -vertex of $P(p, q, r)$. In this case, if $k$ is fixed, then we can find that

$$
\max \left\{d_{u}+m_{u} \mid u \in V(G)\right\}=k+3+\frac{k+7}{k+3}=k+4+\frac{4}{k+3} .
$$

By Lemma 2.1, we have

$$
q_{1}\left(G^{*}\right)<k+4+\frac{4}{k+3} \leq n-\frac{3 g+1}{2}+4+\frac{4}{n-\frac{3 g+1}{2}+3} \leq n-\frac{3 g+1}{2}+5
$$

since the function $k+4+\frac{4}{k+3}$ is increasing with the nonnegative number $k$ and $k \leq n-\frac{3 g+1}{2}$. However, $q_{1}\left(P_{\frac{g-1}{2}, \frac{n g+1}{2}, \frac{g+1}{2}}^{n-1}\right)>\Delta+1=n-\frac{3 g+1}{2}+5>q_{1}\left(G^{*}\right)$, a contradiction.

Fact 2. Let $G^{*}$ have the maximal signless Laplacian spectral radius among all graphs in $\mathcal{B}_{2}(n, g)$, when $n \geq\lceil 3 g / 2\rceil-1$. Then $G^{*} \cong P_{\lfloor g / 2\rfloor,\lceil g / 2\rceil,\lceil g / 2\rceil}^{n-\lceil 3 g / 2\rceil+1}$ and $q_{1}\left(G^{*}\right)<n-\lceil 3 g / 2\rceil+5+\frac{4}{n-\lceil 3 g / 2\rceil+4}$. 
Proof of Fact 2. By Fact 1, we have $G^{*}$ is obtained from by attaching $n-\lceil 3 g / 2\rceil+1$ pendent edges to a unique vertex $u$ of $P(\lfloor g / 2\rfloor,\lceil g / 2\rceil,\lceil g / 2\rceil)$. Next we only need to prove that $u$ is a 3 -vertex of $P(\lfloor g / 2\rfloor,\lceil g / 2\rceil,\lceil g / 2\rceil)$. Assume to contrary that $u$ is a 2-vertex of $P(\lfloor g / 2\rfloor,\lceil g / 2\rceil,\lceil g / 2\rceil)$. For convenience, set $k=n-\lceil 3 g / 2\rceil+1$. If $k=0$, clearly $G^{*} \cong P_{\lfloor g / 2],\lceil g / 2\rceil,\lceil g / 2\rceil}^{0}$.
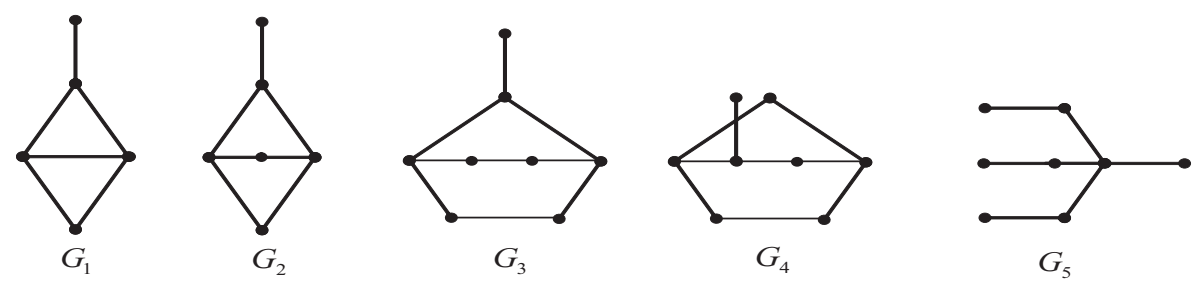

Figure 3: Several special graphs.

Now we consider the case $k=1$. If $g \in\{3,4,5\}$, then $G^{*}$ is isomorphic to one of $G_{i}$ $(i \in\{1,2,3,4\}$, see Figure 3$)$. Straightforward calculations show that $q_{1}\left(G_{1}\right)=5.4679<$ $q_{1}\left(P_{1,2,2}^{1}\right)=5.7785, q_{1}\left(G_{2}\right)=5.2361<q_{1}\left(P_{2,2,2}^{1}\right)=5.5141, \max \left\{q_{1}\left(G_{3}\right), q_{1}\left(G_{4}\right)\right\}=$ $\max \{5.0664,4.9891\}<q_{1}\left(P_{2,3,3}^{1}\right)=5.3552$, a contradiction. If $g \geq 6$, then $u$ can not be simultaneously adjacent to the two 3 -vertices of $P(\lfloor g / 2\rfloor,\lceil g / 2\rceil,\lceil g / 2\rceil)$ and the two 3 -vertices are not adjacent. So $d_{u} m_{u} \leq 1+2+3=6$. Besides, $d_{v_{i}} m_{v_{i}} \leq 2+2+3=7$ if $v_{i}$ is a 3-vertex different from $u, d_{v_{i}} m_{v_{i}} \leq 3+3=6$ if $v_{i}$ is a 2-vertex and $d_{v_{i}} m_{v_{i}}=3$ if $v_{i}$ is a pendent vertex. This implies that

$$
\max \left\{\frac{d_{u}\left(d_{u}+m_{u}\right)+d_{v}\left(d_{v}+m_{v}\right)}{d_{u}+d_{v}} \mid u v \in E\left(G^{*}\right)\right\} \leq \max \left\{\frac{31}{6}, \frac{26}{5}, \frac{20}{4}\right\}=\frac{26}{5},
$$

since $d_{u}+d_{v} \in\{4,5,6\}$ for each edge $u v \in E(G)$. By Lemma 2.7, we have $q_{1}\left(G^{*}\right) \leq \frac{26}{5}$. However, when $g \geq 6, G_{5}$ is a subgraph of $P_{\lfloor g / 2\rfloor,\lceil g / 27,\lceil g / 2\rceil}^{1}$. Thus $q_{1}\left(P_{\lfloor g / 2\rfloor,\lceil g / 2],\lceil g / 2\rceil}^{1}\right)>$ $q_{1}\left(G_{5}\right)=3+\sqrt{5}>\frac{26}{5}$. This is, $q_{1}\left(G^{*}\right)<q_{1}\left(P_{\lfloor g / 2\rfloor,\lceil g / 2],\lceil g / 2\rceil}^{1}\right)$, a contradiction. Also we can consider another more simpler method. When $k=1$, we observe that any maximal graph $G$ on girth $g \geq 6$ by Lemma 2.2 has a smaller signless Laplacian spectral radius than that of the maximal graph of girth $g=5$, namely $G_{3}$, that is $q_{1}(G)<q_{1}\left(G_{3}\right)=5.0664<\frac{26}{5}$ by Lemma 2.3. So the result is also proved.

Next we consider the case $k \geq 2$. We have that $\max \left\{d_{u}+m_{u} \mid u \in V\left(G^{*}\right)\right\}$ attains the maximal value just when $u$ is simultaneously adjacent to the two 3 -vertices of $P(\lfloor g / 2\rfloor,\lceil g / 2\rceil,\lceil g / 2\rceil)$. In this case,

$$
\max \left\{d_{u}+m_{u} \mid u \in V\left(G^{*}\right)\right\}=k+2+\frac{k+6}{k+2} \leq k+4
$$

since $k \geq 2$. By Lemma 2.7, we have $q_{1}\left(G^{*}\right)<k+4$ since $G^{*}$ is neither regular nor semiregular. However, by Lemma 2.6, $q_{1}\left(P_{\lfloor g / 2\rfloor,\lceil g / 2\rceil,\lceil g / 2\rceil}^{n-\lceil 3 / 27\rceil 1}\right)>\Delta+1=k+4$ since $P_{\lfloor g / 2],\lceil g / 2\rceil,\lceil g / 2\rceil}^{n-\lceil 3 g / 2\rceil+1}$ is not a star. Note that $P_{\lfloor g / 2\rfloor,[g / 2\rceil,\lceil g / 2\rceil}^{n-\lceil 3 / 2\rceil+1}$ is neither regular nor semi-regular bipartite except 
that $P_{2,2,2}^{0} \cong K_{2.3}$ is a semi-regular bipartite graph. Thus if $(n, g) \neq(5,4)$,

$q_{1}\left(P_{\lfloor g / 2\rfloor,\lceil g / 2\rceil,\lceil g / 2\rceil}^{n-\lceil 3 g / 2\rceil+1}\right)<\max \left\{d_{u}+m_{u} \mid u \in V\left(P_{\lfloor g / 2\rfloor,\lceil g / 2\rceil,\lceil g / 2\rceil}^{n-\lceil 3 g / 2\rceil+1}\right)\right\} \leq n-\left\lceil\frac{3 g}{2}\right\rceil+5+\frac{4}{n-\left\lceil\frac{3 g}{2}\right\rceil+4}$

since $k+3+\frac{4}{k+2}$ is increasing with nonnegative integer $k$.

As for $(n, g)=(5,4)$, we know that

$$
q_{1}\left(P_{2,2,2}^{0}\right)=5<\frac{16}{3}=n-\left\lceil\frac{3 g}{2}\right\rceil+5+\frac{4}{n-\left\lceil\frac{3 g}{2}\right\rceil+4} .
$$

Theorem 3.4 Let $G^{*}$ have the maximal signless Laplacian spectral radius among all graphs in $\mathcal{B}(n, g)$, where $n \geq\lceil 3 g / 2\rceil-1$. Then $G^{*} \cong P_{\lfloor g / 2\rfloor,\lceil g / 2\rceil,\lceil g / 2\rceil}^{n-\lceil 3 g / 2\rceil+1}$ and $q_{1}\left(P_{\lfloor g / 2\rfloor,\lceil g / 2\rceil,\lceil g / 2\rceil}^{n-\lceil 3 g / 2\rceil+1}\right)<n-\lceil 3 g / 2\rceil+5+\frac{4}{n-\lceil 3 g / 2\rceil+4}$.

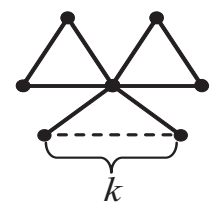

$B_{3,3}^{n-5}$

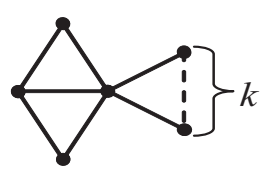

$P_{1,2,2}^{n-4}$

Figure 4: Graphs $B_{3,3}^{n-5}$ and $P_{1,2,2}^{n-4}$.

Proof. We distinguish the two cases as follows:

Case 1. If $g=3$, the corresponding graphs $B_{3,3}^{n-5}$ and $P_{1,2,2}^{n-4}$ are shown in Figure 4, then by Lemma 2.2, we have $q_{1}\left(B_{3,3}^{n-5}\right)<q_{1}\left(P_{1,2,2}^{n-4}\right)$.

Case 2. If $g \geq 4$, we have to show $q_{1}\left(B_{g, g}^{n-2 g+1}\right)<q_{1}\left(P_{\lfloor g / 2\rfloor,\lfloor g / 2\rfloor,\lfloor g / 2\rfloor}^{n-\lceil g g / 2\rceil+1}\right)$. According to the results above, $q_{1}\left(B_{g, g}^{n-2 g+1}\right)<n-2 g+6+\frac{4}{n-2 g+5} \leq n-2 g+7$ since in this case $n \geq 2 g-1$. However $q_{1}\left(P_{\lfloor g / 2\rfloor,\lfloor g / 2\rfloor,\lfloor g / 2\rfloor}^{n-\lceil 3 / 2\rceil+1}\right)>\Delta+1=n-\lceil 3 g / 2\rceil+5$. Since $g \geq 4$, $n-\lceil 3 g / 2\rceil+5-(n-2 g+7)=\lfloor g / 2\rfloor-2 \geq 0$.

This completes the whole proof of Theorem 3.4.

\section{Acknowledgements}

The authors would like to express their thanks to an anonymous referee for many detailed comments and suggestions, which are very helpful for improving the presentation of the manuscript. 


\section{References}

[1] M. Aouchiche, P. Hansen, A survey of automated conjectures in spectral graph theory, Linear Algebra Appl., 432(2009), 2293-2322.

[2] D. Cvetković, P. Rowlinson, S. K. Simić, Eigenvalue bounds for the signless Laplacian, Publ. Inst. Math. (Beograd), 81(95)(2007), 11-27.

[3] D. Cvetković, S. K. Simić, Towards a spectral theory of graphs based on the signless Laplacian, I, Publ. Inst. Math. (Beograd), 85(99)(2009), 19-33.

[4] D. Cvetković, S. K. Simić, Towards a spectral theory of graphs based on the signless Laplacian, II, Linear Algebra Appl., 432(2010), 2257-2272.

[5] D. Cvetković, S. K. Simić, Towards a spectral theory of graphs based on the signless Laplacian, III, Appl. Anal. Discrete Math., 4(2010), 156-166.

[6] L. H. Feng, The signless Laplacian spectral radius for bicyclic graphs with $k$ pendant vertices, Kyungpook Math.J., 50(2010), 100-116.

[7] L. H. Feng, G. H. Yu, The signless Laplacian spectral radius of unicyclic graphs with graph constraints, Kyungpook Math. J., 49(2009), 123-131.

[8] L. Feng, Q. Li, X. D. Zhang, Minimizing the Laplacian spectral radius of trees with given matching number, Linear Multilinear Algebra, 55(2)(2007), 199-207.

[9] Y. Z. Fan, D. Yang, the signless Lapalcian spectral radius of graphs with given number of pendent vertices, Graphs and Combinatorics., 25(3)(2009), 291-298.

[10] L. H. Feng, G. H. Yu, A. Ilić, The Laplacian spectral radius for unicyclic graphs with given independence number, Linear Algebra Appl., 433(5)(2010), 934-944.

[11] Y. Hong, X.D. Zhang, Sharp upper and lower bounds for largest eigenvalue of the Laplacian matrix of trees, Discrete Math., 296(2-3)(2005), 187-197.

[12] M. H. Liu, X. Z. Tan, B. L. Liu, The (signless) Laplacian spectral radius of unicyclic and bicyclic graphs with $n$ vertices and $k$ pendant vertices, Czechoslovak Mathematical J., 60(135)(2010), 849-867.

[13] C. S. Oliverira, L. S. Lima, N. M. M. Abreu, P. Hansen, Bounds on the index of the signless Laplacian of a graph, Discrete Appl Math., 158(4)(2010), 355-360.

[14] X. D. Zhang, The Laplacian eigenvalues of graphs: a survey, In: Linear Algebra Research Advances, Editor: Gerald D. Ling, Nova Science Publishers, Inc., 2007, $201-228$.

[15] M. Q. Zhai, G. L. Yu, J. L. Shu, the Laplacian spectral radius of bicyclic graphs with a given girth, Computers and Mathematics with Applications., 59(1)(2010), 376-381. 